\section{Kidney \\ Blood Pressure Research}

\title{
High Salt Intake Increases Blood Pressure in Normal Rats: Putative Role of 20-HETE and No Evidence on Changes in Renal Vascular Reactivity
}
A. Walkowska ${ }^{a}$
M. Kuczeriszka ${ }^{a}$
J. Sadowskia
K.H. Olszyńskia
L. Dobrowolskia
L. Červenkab
B.D. Hammock ${ }^{c}$
E. Kompanowska-Jezierska ${ }^{a}$

a Department of Renal and Body Fluid Physiology, M. Mossakowski Medical Research Centre, Polish Academy of Sciences, Warsaw, Poland; ${ }^{b}$ Center for Experimental Medicine, Institute for Clinical and Experimental Medicine, Prague, Czech Republic: 'Department of Entomology and UCD Comprehensive Cancer Center, University of California, Davis, California, USA

\section{Key Words}

High salt diet $•$ Hypertension $\bullet 20-$ HETE

\begin{abstract}
Background/Aims. High salt (HS) intake may elevate blood pressure (BP), also in animals without genetic salt sensitivity. The development of salt-dependent hypertension could be mediated by endogenous vasoactive agents; here we examined the role of vasodilator epoxyeicosatrienoic acids (EETs) and vasoconstrictor 20-hydroxyeicosatetraenoic acid (20HETE). Methods. In conscious Wistar rats on HS diet systolic BP (SBP) was examined after chronic elevation of EETs using 4-[4-(3-adamantan-1-yl-ureido)-cyclohexyloxy]-benzoic acid (c-AUCB), a blocker of soluble epoxide hydrolase, or after inhibition of 20-HETE with 1 -aminobenzotriazole (ABT). Thereafter, in acute experiments the responses of renal artery blood flow (Transonic probe) and renal regional perfusion (laser-Doppler) to intrarenal acetylcholine $(\mathrm{ACh})$ or norepinephrine were determined. Results. HS diet increased urinary 20-HETE excretion. The SBP increase was not reduced by c-AUCB but prevented by ABT until day 5 of HS exposure. Renal vasomotor responses to $A C h$ or norepinephrine were similar on standard and HS diet. ABT but not $c-A \cup C B$ abolished the responses to ACh. Conclusions. 20-HETE seems to mediate the early-phase HS diet-induced BP increase while EETs are not engaged in the process. Since HS exposure did not alter renal vasodilator responses to Ach, endothelial dysfunction is not a critical factor in the mechanism of salt-induced blood pressure elevation.
\end{abstract}




\section{Kidney Blood Pressure Research}

Kidney Blood Press Res 2015;40:323-334

DOI: 10.1159/000368508

Published online: May 31, 2015

(C) 2015 S. Karger AG, Basel

www.karger.com/kbr

\section{Introduction}

Vast evidence indicates that salt excess has an important role in the pathogenesis of many forms of human and experimental hypertension [1-4]; high salt intake is also known to induce functional and structural changes in the vasculature that are not dependent on blood pressure (BP) elevation per se [5] while salt restriction was found to act in the opposite direction $[6,7]$. Over the years, a considerable amount of research accumulated indicating the involvement of the renin-angiotensin system (RAS) in the development of salt-dependent hypertension [8]. On the other hand, despite long-lasting research the actual role of active products of cytochrome P-450 (CYP-450) dependent arachidonic acid (AA) monooxygenases and interaction of these products with nitric oxide (NO) cascade remain unclear $[9,10]$.

The active AA metabolites have been implicated in regulation of the vascular tone and arterial pressure $[9,11,12]$. They can influence BP directly, by altering the vessel tone: most of epoxyeicosatrienoic acids (EETs), generated by epoxygenase, induce relaxation whereas 20-hydroxyeicosatetraenoic acid (20-HETE), the product of $\omega$-hydroxylase, is a vasoconstrictor [11]. On the other hand, both EETs and 20-HETE inhibit renal tubular reabsorption: the consequent increase in renal excretion, when sufficiently long-lasting, may result in depletion of body fluids and a decrease in blood pressure. Augmenting EETs activity by inhibition of their degradation, as obtained using cis-4-[4-(3-adamantan-1-yl-ureido)cyclohexyloxy]-benzoic acid (c-AUCB), was recently reported to attenuate the increase in BP in hypertensive Ren-2 transgenic rats [13]. The mechanism of these effects is complex but certainly involves interaction of the two agents with the NO cascade $[9,10]$.

There is evidence that renal content and activity of CYP-450 dependent metabolites of AA are differentially modified by $\mathrm{NaCl}$ intake. Explicitly, high dietary salt downregulates the expression of CYP4A which is engaged in generation of 20-HETE in kidney tissue and renal vasculature [10]. In contrast, formation of EETs in the kidney is increased by high-salt diet [14]. This response is typical for salt-resistant animals and is not associated with an increase in BP. Increased dietary salt intake was reported to interfere with normal NO release and the vasorelaxation in response to vasodilator stimuli [15-17]. It will be noticed that high sodium intake stimulates the synthesis of NO but its bioavailability may be reduced as a consequence of increased production of reactive oxygen species (ROS) [18]. This suggests that salt-induced dysfunction of the vascular endothelium is probably induced by local oxidative stress [19].

Our aim was to examine if the modest BP elevation observed in essentially saltresistant normotensive Wistar rats after exposure to high salt intake could be at least in part mediated by alterations in the availability and/or action of EETs and 20-HETE. Such mediation appeared plausible since, as discussed above, sodium overload could alter CYP450 metabolic pathways and/or NO synthesis and affect BP, e.g. by altering the blood vessel reactivity to vasoactive agents. Of special interest would be alterations within the renal vascular bed where, aside from the effects of changing vascular resistance per se, specific modifications of circulation in the renal medulla could alter tubular reabsorption and renal excretion and cause fluid volume dependent changes in BP.

To explore the putative contribution of changes in CYP-450 dependent AA metabolism in rats on high-sodium diet the generation of 20-HETE was inhibited using 1-aminobenzotriazole (ABT). At the dose applied here it is a potent inhibitor of renal CYP-450 dependent $\omega$-hydrolases with less effect on epoxygenases [20,21]. EETs content was raised by inhibition of soluble epoxygenase hydrolase ( $\mathrm{SEH}$ ) which normally degrades EETs to relatively inactive dihydroxyepoxytrieonic acids (DHETEs). The renal vascular responses to vasodilator acetylcholine (Ach) or vasoconstrictor norepinephrine (NE) were tested. The former response could expose a possible role of the functional status of the endothelium; to further explore this possibility, intrarenal NO activity was also determined. To examine the possibility that some selective alterations of the renal medullary circulation are crucial for the increase in BP [22], in addition to determination of total RBF the perfusion of the renal cortex and outer and inner medulla was separately measured. 


\section{Kidney Blood Pressure Research}

Walkowska/Kuczeriszka/Sadowski/Olszyński/Dobrowolski/Červenka/Hammock/ Kompanowska-Jezierska: 20-HETE in Salt Induced BP Increase in Rats

\section{Materials and Methods}

The experimental procedures were approved by the Extramural First Ethical Committee for Animal Experimentation, Warsaw, whose regulations conform to the provisions of the Declaration of Helsinki, 1995. Male Wistar rats weighing $306 \pm 2 \mathrm{~g}$ had free access to food and tap water until the day of an acute experiment.

Before an acute experiment rats were kept on standard diet (STD, 0.25\% Na w/w) or on high-sodium $\operatorname{diet}(\mathrm{HS}, 4 \% \mathrm{Na}, \mathrm{w} / \mathrm{w}$, SSNIFF GmbH, Soest, Germany) for 10 days. Over this period blood pressure (tail cuff method, CODA, Kent Scientific, USA) was measured on days 0, 2, 5 and 9, always at the same time of the day (around 10:00- 12:00 a.m.).

During the 10 days preceding the start of experimental measurements the rats were accustomed to the restraint needed for BP measurement. Rats fed HS diet were untreated or pretreated with cis-4-[4-(3adamantan-1-yl-ureido)-cyclohexyloxy]-benzoic acid (c-AUCB) a soluble epoxide hydrolase (sEH) inhibitor [23]. c-AUCB was given in drinking water at a concentration of $26 \mathrm{mg} / \mathrm{l}$ which provided $17 \mathrm{mg} / \mathrm{kg} / \mathrm{day}$. This was the highest dose used in recent studies and it was reported to substantially increase tissue EETs concentration [17]. In another HS group, 1-aminobenzotriazole (ABT, Sigma-Aldrich, Basel, Switzerland), an inhibitor of CYP-450 monooxygenases was given at $50 \mathrm{mg} / \mathrm{kg} /$ day; at this dosage ABT was shown to have only little effect on epoxygenases [10, 14]. For the seven final days of HS diet, c-AUCB was given in drinking water, and ABT was given as intraperitoneal injections. After chronic part of the study, in terminal acute experiments under anaesthesia the rats received, via renal artery, acetylcholine (ACh, Sigma, Basel, Switzerland) or norepinephrine (NE, Sigma-Aldrich, Basel, Switzerland), to evaluate the reactivity of the renal circulation to vasoconstrictor or vasorelaxant agents.

In additional studies we determined urinary 20-HETE concentration in freely moving rats maintained on STD or HS diet for 21 days. Urine samples were collected one day before the switch from STD to HS diet (day 0) and later on days: 2, 7, 14 (data shown in Table 1) and also on day 21 of HS exposure, to be compared with samples from rats on continued STD diet. In the same samples urine osmolality (Uosm) and sodium concentration (UNa) were measured. Urine 20-HETE concentrations were standardized to the corresponding Uosm.

\section{Surgical preparations}

Rats were anaesthetized with intraperitoneal sodium thiopental (Sandoz GmbH, Kundl, Austria), $100 \mathrm{mg} / \mathrm{kg}$, which provided stable anaesthesia for at least $4 \mathrm{~h}$. In HS rats the initial dose of thiopental was reduced by one-third, because of increased sensitivity of these animals to the drug. The rats were placed on a heated surgery table to maintain rectal temperature at about $37^{\circ} \mathrm{C}$. A polyethylene tube was placed in the trachea to ensure free airways.

The jugular vein was cannulated for fluid infusions, the femoral artery for blood sampling and the carotid artery for mean arterial blood pressure (MABP) measurement (Stoelting blood pressure meter and transducers, Wood Dale, Illinois, USA). During surgery, 3\% bovine serum albumin solution was infused i.v. at $3 \mathrm{ml} / \mathrm{h}$ to preserve plasma volume. The left kidney was exposed from a subcostal flank incision and placed in a plastic holder, similar as that used for micropuncture. The ureter was cannulated for timed urine collection.

The total renal blood flow (RBF) was measured using a non-cannulating probe, $1 \mathrm{~mm}$ in diameter, placed on the renal artery and connected with a Transonic flowmeter (type T106; Transonic System, Ithaca, NY, USA). A laser-Doppler probe, type PF 407, was placed on kidney surface to record the superficial cortical blood flow (CBF). The outer medullary blood flow (OMBF) and the inner medullary blood flow (IMBF) were measured as laser-Doppler fluxes using two needle probes (PF 402) inserted to the respective depths of 3 and $5 \mathrm{~mm}$ from kidney surface. The probes were connected with a Periflux 5010 flowmeter (Perimed, Jarfalla, Sweden). They were calibrated using a motility standard (a colloidal suspension of latex particles). The Brownian motion of the suspension provides the standard value of 250 perfusion units ( $1000 \mathrm{PU}=10 \mathrm{mV}$ ). Thus, only relative flux values were measured but the calibration enabled comparison of the results between animals. At the end of experiments the positioning of the probes was examined at the kidney's cross-section. Since, in our opinion, the above calibration and verification of the positioning of the probes did not entirely eliminate the uncertainty involved in comparing the values between groups (in contrast to time-dependent 


\section{Kidney Bloód Pressure Research}

changes in the same animal), only the most robust differences in medullary flow values were considered and interpreted.

An L-shaped needle was inserted via aorta into the renal artery for intrarenal artery infusion of ACh and NE. Changes in $\mathrm{RBF}, \mathrm{CBF}, \mathrm{OMBF}$ and IMBF in response to the two vasoactive substances were used as a measure of intrarenal vascular reactivity in individual kidney zones.

\section{Protocols of acute experiments}

At the end of surgical preparation and after placement of the flow probes, 20-min urine collections were made to determine baseline diuresis and sodium and total solute excretion in each of four groups. After stabilization of renal haemodynamics, acetylcholine (ACh, 5 or $10 \mu \mathrm{g} / \mathrm{kg} / \mathrm{h}$ ) or norepinephrine (NE, 10 or $30 \mu \mathrm{g} / \mathrm{kg} / \mathrm{h}$ ) was infused during 10 minutes, at a random order, directly into the renal artery, to avoid effects on systemic blood pressure. Afterwards, the usual saline infusion was restored and, after stabilization, the infusion of the other dose of ACh or NE was repeated. Since there were no differences between the responses to the two doses of ACh or NE, the data were pooled. This basic protocol was applied in four experimental groups:

1. Untreated rats maintained on standard diet (STD, $n=6)$

2. Untreated rats maintained on high sodium diet (HS, $\mathrm{n}=7$ )

3. $c$-AUCB pretreated rats $(17 \mathrm{mg} / \mathrm{kg} /$ day), maintained on HS diet $(\mathrm{HS}+c-A U C B, \mathrm{n}=6)$

4. ABT pretreated rats $(50 \mathrm{mg} / \mathrm{kg}$ i.p., dissolved in $0.5 \mathrm{ml}$ isotonic saline), maintained on $\mathrm{HS}$ $\operatorname{diet}(\mathrm{HS}+\mathrm{ABT}, \mathrm{n}=6)$

Four additional experimental groups were studied to evaluate nitric oxide bioavailability in kidney tissue as affected by HS diet and/or ABT pretreatment. Wistar male rats were kept on standard or high sodium diet for 21 days, and on the two last days preceding an acute experiment they received an intraperitoneal injection of ABT (50 mg/kg). It was reported earlier that such two-days' treatment was sufficient to effectively inhibit CYP-450 activity [14]. In the final acute experiment MABP, RBF, CBF and MBF (medullary blood flow measured at the border of outer and inner medulla) were determined in rats surgically prepared as described above, in the following groups:

5. Untreated rats maintained on standard diet (STD, $n=9)$,

6. Untreated rats maintained on HS diet (HS21, $n=6)$,

7. ABT pretreated rats maintained on standard diet (STD+ABT, $n=6$ ),

8. ABT pretreated rats maintained on HS diet, (HS21+ABT, $n=5)$

For measurement of the renal medullary NO signal, a needle-shaped ISO-NOP 200 sensor $(0.2 \mathrm{~mm}$ in diameter), connected with Nitric Oxide Meter (ISO-NO MARK II, World Precision Instruments, Inc., USA), was inserted to the depth of $5 \mathrm{~mm}$. To verify in vitro the responsiveness of the sensor, calibration curves relating the readings $(\mathrm{pA})$ to known increasing concentrations of NO released from S-Nitroso-N-acetyl-D,L-penicillamine (SNAP) were established as recommended by the manufacturer of the equipment and described in detail by Zhang \& Broderick [24]. The procedure is based on the decomposition of SNAP in the presence of a catalyst, $\mathrm{Cu}(\mathrm{I})$, leading to a release of NO. The results of studies in vivo were expressed in pA. In vivo tests confirmed a dose-dependent decrease in tissue NO signal in response to intravenous administration of L-NAME, and an increase in NO after renal artery infusion of SNAP, in agreement with earlier reports from this laboratory [25]. 


\section{Kidney Blood Pressure Research}

Analytical procedures

Urine osmolality was determined by freezing-point depression using a semi-micro osmometer (Osmomat 030, Gonotec, Germany) and sodium concentrations by flame photometer (Jenway PFP7, Essex, UK). 20-HETE concentration in urine samples was measured by gas chromatography (Shimadzu GC-17A, Shimadzu, Japan) using own calibration standards prepared from synthetic 20-HETE (Sigma, USA).

\section{Statistics}

Data are expressed as means \pm SEM. Significance of changes within one group over time was first evaluated by repeated measures analysis of variance (ANOVA; STATISTICA, version 10, StatSoft Inc.), followed by Student $\mathrm{t}$ test for dependent variables. Differences between groups were first analyzed by the classical one-way ANOVA followed by two-sided modified Student t-test for independent variables, using Bonferroni correction for multiple comparisons. $P \leq 0.05$ was taken to indicate significance of differences.

\section{Results}

Fig. 1 shows changes in systolic blood pressure (SBP, tail-cuff method) in conscious rats. Throughout nine days of observation SBP remained stable in rats on standard diet. In the untreated group maintained on HS diet, SBP increased progressively; it was significantly elevated beginning from the day 5 of the $\operatorname{diet}(165 \pm 4$ vs. $146 \pm 5 \mathrm{mmHg}, P=0.03$ ). The whole profile seen in untreated HS rats was significantly different from that observed in the STD group (repeated measurements ANOVA, $\mathrm{p}<0.05$ ).

In HS rats pretreated with $c$-AUCB, over the first 5 days SBP increased in parallel with the increase in untreated HS rats, thereafter, however, a further increase in SBP was seen whereas the pressure remained stable in the untreated HS group (Fig. 1). Pretreatment of HS rats with ABT delayed the increase in SBP: on day 5 of the exposure to HS diet SBP was still at the control level. On day 9, it was significantly above control (+ 10\%). Remarkably, after 21 days' exposure to high salt diet the rats pretreated with ABT showed slightly lower (NS) BP when compared to untreated animals (Fig. 4). The stimulatory action of HS diet on generation of 20-HETE was verified by determination of the agent's concentration in urine (Table 1). The data show that in rats on standard diet the levels were stable over two weeks whereas in HS rats an increase was seen already on day 2 of high salt intake; beginning from day 7 , the urinary 20-HETE was substantially and significantly elevated compared with that measured in STD rats. We checked also that the elevation was maintained when measured on day 21 of exposure to HS diet when the value was $1.08 \pm 0.14$ [(nmol/osmol)*10].

To evaluate the reactivity of intrarenal vessels to vasoactive agents, in terminal acute experiments performed after chronic treatments the rats were given renal artery infusions of acetylcholine $(\mathrm{ACh})$ or norepinephrine (NE). Baseline values of mean arterial blood maintained on standard (STD) or high (HS) sodium diet, untreated or pretreated with ABT (1-aminobenzotriazole) or $c$-AUCB (cis-4[4-(3-adamantan-1-yl-ureido)-cyclohexyloxy]-benzoic acid. Means \pm SEM, * significantly different from day 0 , † significantly different from STD, \# significantly different from HS; $p<0.05$. 


\section{Kidney \\ Blood Pressure Research}

Table 2. Basal values of mean arterial blood pressure (MABP), total renal blood flow (RBF) and laser Doppler fluxes reflecting perfusion of the cortex (CBF), outer medulla (OMBF) and inner medulla (IMBF), and of urine flow $(\mathrm{V})$ and sodium excretion ( $\mathrm{UNaV}$ ), measured in acute experiments with acetylcholine and norepinephrine injections

\begin{tabular}{lccccccc}
\hline & MABP & RBF & CBF & OMBF & IMBF & V & $\mathrm{U}_{\mathrm{NaV}}$ \\
\cline { 2 - 7 } & $\mathrm{mmHg}$ & $\mathrm{ml} / \mathrm{min}$ & $\mathrm{PU}$ & $\mathrm{PU}$ & $\mathrm{PU}$ & $\mu \mathrm{l} / \mathrm{min}$ & $\mu \mathrm{mol} / \mathrm{min}$ \\
\hline STD $(\mathrm{n}=6)$ & $104 \pm 2$ & $7.5 \pm 0.7$ & $585 \pm 41$ & $201 \pm 28$ & $173 \pm 20$ & $4.4 \pm 1.0$ & $0.13 \pm 0.01$ \\
HS $(\mathrm{n}=7)$ & $112 \pm 5$ & $6.5 \pm 1.7$ & $625 \pm 79$ & $136 \pm 19$ & $152 \pm 18$ & $9.6 \pm 3.6^{*}$ & $2.69 \pm 1.21^{*}$ \\
HS+AUCB $(\mathrm{n}=6)$ & $114 \pm 7$ & $9.5 \pm 3.1$ & $678 \pm 78$ & $231 \pm 42 \dagger$ & $135 \pm 30$ & $10.2 \pm 1.6$ & $2.42 \pm 0.51$ \\
HS+ABT $(\mathrm{n}=6)$ & $95 \pm 4 \dagger$ & $8.9 \pm 1.5$ & $574 \pm 50$ & $177 \pm 34$ & $124 \pm 20$ & $8.6 \pm 2.7$ & $1.55 \pm 0.71$ \\
\hline
\end{tabular}

STD, HS - untreated rats on standard or high-sodium diet; HS+AUCB, HS+ABT - rats on high-sodium diet treated with soluble epoxide hydrolase inhibitor (AUCB) or CYP-450 inhibitor 1-aminobenzotriazol (ABT). PU - perfusion units; Means \pm SEM, * significantly different from untreated STD rats, $\uparrow$ significantly different from untreated STD rats.

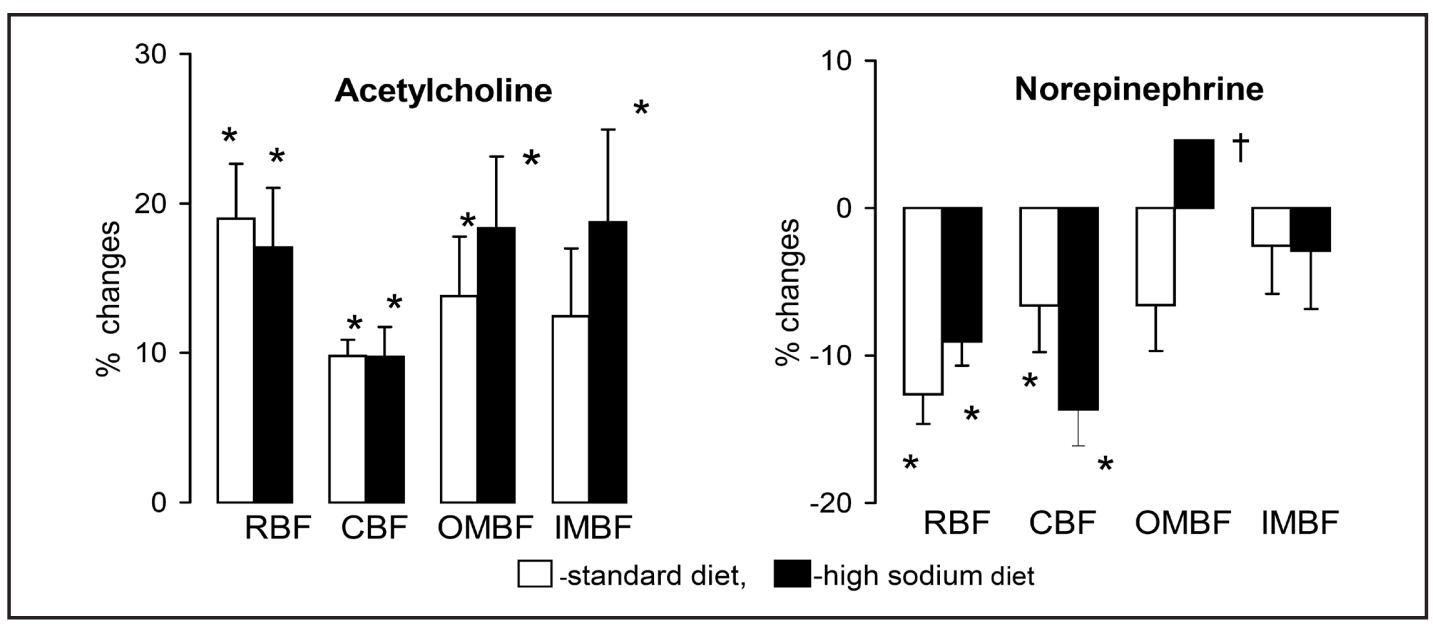

Fig. 2. Changes in total renal blood flow (RBF) and flows through the cortex (CBF) and outer (OMBF) and inner medulla (IMBF) in response to acetylcholine and norepinephrine infused into renal artery. The data for day 10 of exposure to diets. Means \pm SEM, * significantly different from pre-infusion control, $†$ significantly different from standard diet.

pressure (MABP), total renal blood flow (RBF), and laser-Doppler fluxes reflecting perfusion of the cortex, outer- and inner medulla (CBF, OMBF, IMBF) as well as $\mathrm{V}$ and $\mathrm{UNaV}$ are shown in Table 2. MABP were usually higher in HS groups compared to rats on standard diet with the exception that HS ABT-treated rats showed a value significantly lower than the HS untreated animals. Of interest was also a comparison of OMBF values: under high salt intake mean OMBF value was more than 30\% lower than in the STD group. On the other hand, that measured in HS rats treated with $c$-AUCB was about $70 \%$ higher $(\mathrm{p}<0.05)$ than without treatment. As expected, exposure to HS diet distinctly increased $\mathrm{UNaV}$ and $\mathrm{V}$ when compared to the STD group ( $\mathrm{p}<0.05$ for both). $c$-AUCB or ABT treatment did not significantly alter $\mathrm{V}$ or $\mathrm{UNaV}$ in HS rats. The remarkably low pressure in ABT treated rats (the lowest among groups) was probably responsible for the low $\mathrm{UNaV}$ value (44\% lower than in untreated HS controls: "low pressure antinatriuresis").

The vasodilator responses to ACh (a measure of the status of the vascular endothelium) were quite clear in untreated rats irrespective of the diet: both total and regional renal perfusion increased significantly. No significant differences were seen between untreated rat groups on standard (STD) versus high-sodium diet (HS) (Fig. 2). The vasoconstrictor reaction to $\mathrm{NE}$, an index of the responsiveness of the vascular smooth muscle, consisted in significant decreases in RBF and CBF. Again, there were no significant differences in responses to NE depending on the diet. Interestingly, OMBF decreased in the STD but increased in the HS group 


\section{Kidney \\ Blood Pressure \\ Research}

(significant difference in the $\mathrm{r}$ e s p o n s e $\mathrm{s}$, $P<0.04$ ).

The data on the renal haemodynamic responses to ACh and $\mathrm{NE}$ in $\mathrm{HS}$ rats, untreated or pre-treated with $c$-AUCB or ABT, are collected in Fig. 3. The post-ACh increases in renal haemodynamic parameters tended to be modestly reduced under $c$-AUCB treatment and were virtually abolished by ABT. Surprisingly, in response to $\mathrm{ACh}$ the IMBF significantly decreased in the ABT group. The postNE decreases in $\mathrm{RBF}$ and $\mathrm{CBF}$ tended to be smaller and became non-significant in HS rats treated with $c$-AUCB or ABT.

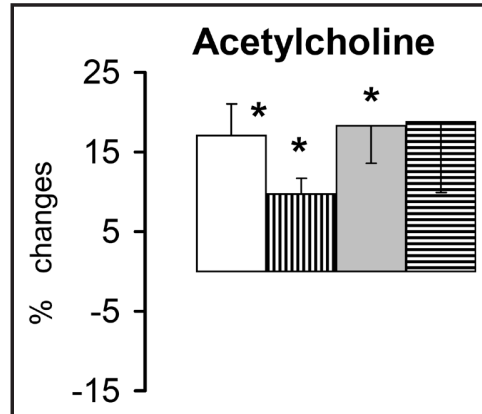
Norepinephrine

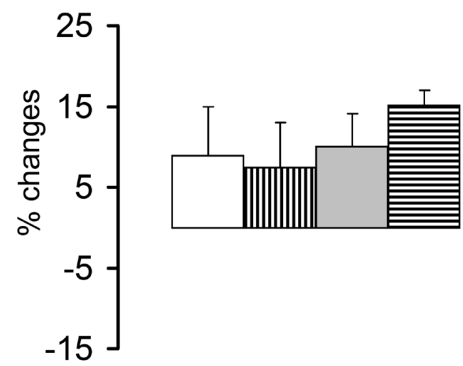

$\%$
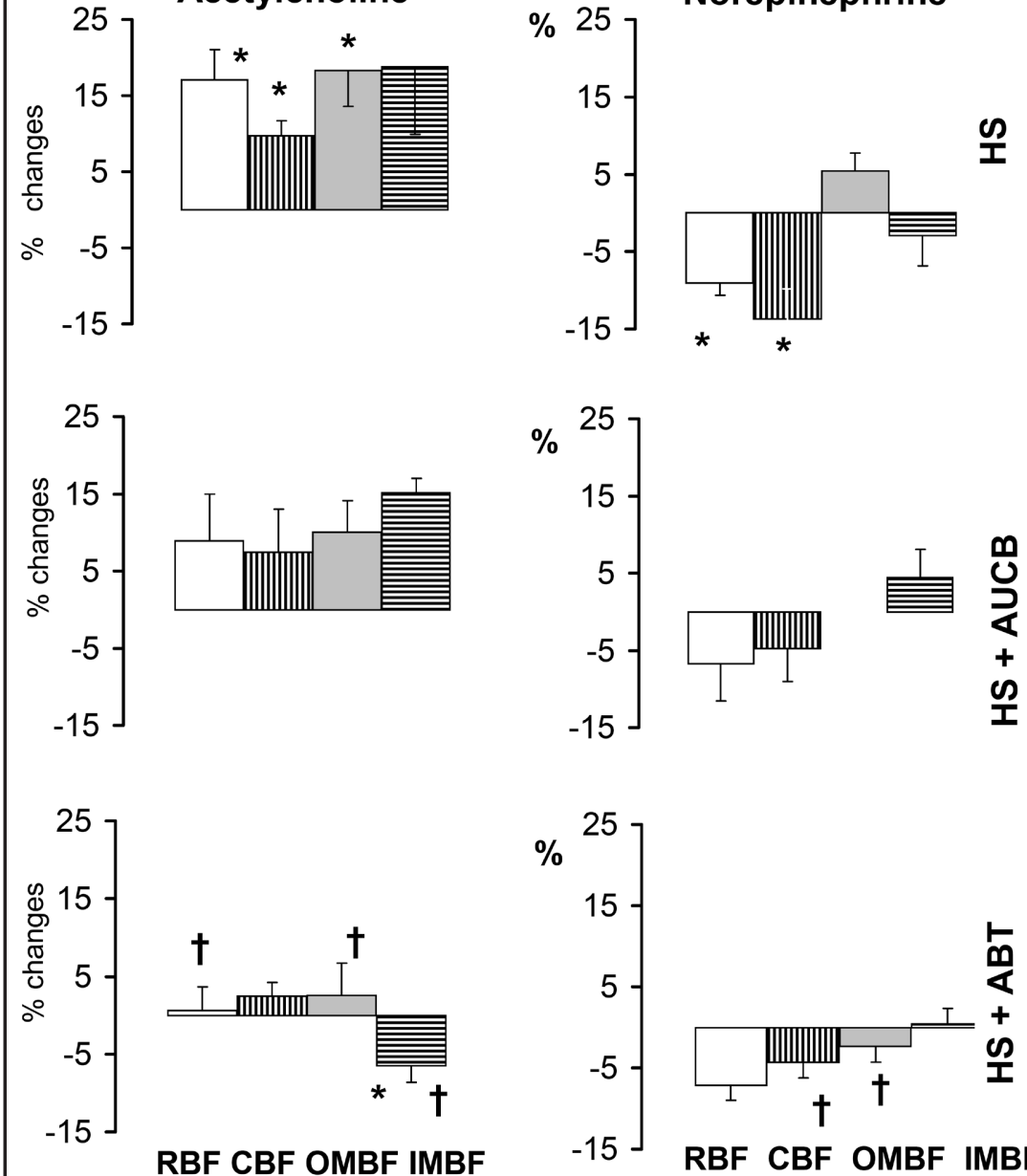

Fig. 3. Changes in total renal blood flow (RBF) and flows through the cortex (CBF) and outer (OMBF) and inner medulla (IMBF) in response to acetylcholine (5 or 10 $\mu \mathrm{g} / \mathrm{kg} / \mathrm{h}$ ) and norepinephrine (10 or $30 \mu \mathrm{g} / \mathrm{kg} / \mathrm{h}$ ) infused into renal artery. HS - high sodium diet, ABT-1-aminobenzotriazole), c-AUCB - cis-4-[4-(3-adamantan-1-ylureido)-cyclohexyloxy]-benzoic acid. The data for day 10 of exposure to diets. Means \pm SEM, * significantly different from pre-infusion control, $\dagger$ significantly different from untreated HS rats.

The changes in medullary perfusion (OMBF, IMBF) were not consistently altered by either treatment, however, NE tended to increase OMBF in untreated HS rats and to decrease it in ABT treated rats.

In rats maintained on HS diet for 21 days MABP, measured at the end of this time period in anaesthetized animals, was significantly higher than in STD rats $(118 \pm 5 \mathrm{vs} .101 \pm 6 \mathrm{mmHg}$; $\mathrm{p}<0.05$ ); the respective difference in rats pretreated with ABT was smaller and not significant (Fig. 4). In ABT pretreated HS rats MBF was significantly higher than without treatment $(232 \pm 24$ vs. $148 \pm 18 \mathrm{PU}, \mathrm{p}<0.02)$. Tissue nitric oxide signal (NO) tended to be higher in STD than in HS rats and in both groups ABT pretreatment induced a significant NO signal elevation (Fig. 4). 


\section{Kidney Blood Pressure Research}

\section{Discussion}

High sodium intake and blood pressure increase: role of cytochrome P450- dependent metabolites of arachidonic acid

A 10 days' exposure of normal Wistar rats to high-sodium intake resulted in this study in an increase in systolic blood pressure (SBP); an elevation was also seen when measured, in another experimental series, in anaesthetized rats after exposure to high-salt diet for 21 days. However, in other studies with rats that were not described as salt-sensitive, an exposure to high salt diet of comparable duration induced no change [26] or only a slight increase in SBP [27, 28]. We hypothesized that the background of salt dependent increase in blood pressure in our studies was some derangement of the metabolism of CYP-450 dependent active agents.

One could expect that experimental alterations of the activity of cytochrome P450-dependent vasoactive and transport-inhibitory metabolites would modify the extent and/or the profile of progressing BP increase in rats exposed to high-salt diet. Indeed, EETs and 20-HETE have an established role in control of BP and kidney function and their effects depend on the sodium intake [9]. High salt intake was commonly reported to increase the activity of the CYP2C isoform and production of EETs in the kidney [29] and to lower renal production of 20-HETE [9, 30]. On the other hand, outside the kidney 20-HETE synthesis was reported to increase in response to high salt intake [31]. In our experimental setting HS diet was found to increase urinary 20-HETE concentration indicating its increased generation in the kidney, a finding supporting our hypothesis of altered activity of CYP-450

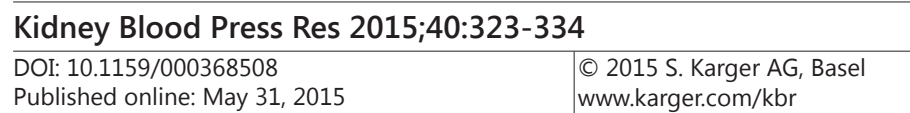

Walkowska/Kuczeriszka/Sadowski/Olszyński/Dobrowolski/Červenka/Hammock/ Kompanowska-Jezierska: 20-HETE in Salt Induced BP Increase in Rats

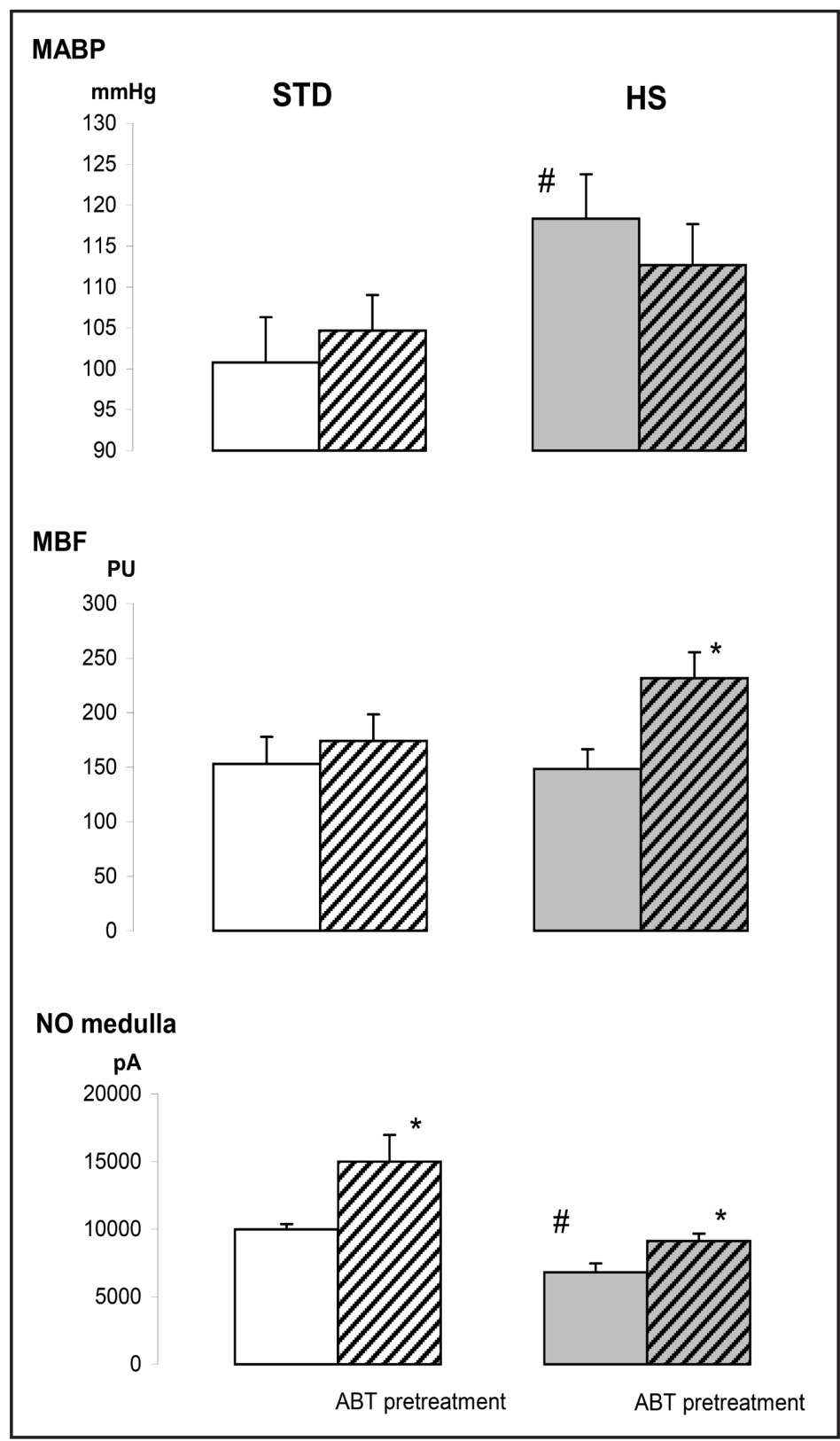

Fig. 4. Effects of high sodium diet (HS) and/or inhibition of CYP-450 dependent monooxygenases (ABT) on mean arterial blood pressure (MABP), medullary blood flow (MBF) and medullary tissue nitric oxide (NO) signal. The data for day 21 of exposure to diet. Means \pm SEM, * significantly different from untreated STD rats, \# significantly different from untreated rats on the same diet. $(\mathrm{p}<0.05, \mathrm{n}=5-9)$. 


\section{Kidney Blood Pressure Research}

Kidney Blood Press Res 2015;40:323-334

DOI: 10.1159/000368508

Published online: May 31, 2015

(c) 2015 S. Karger AG, Basel

www.karger.com/kbr

Walkowska/Kuczeriszka/Sadowski/Olszyński/Dobrowolski/Červenka/Hammock/

Kompanowska-Jezierska: 20-HETE in Salt Induced BP Increase in Rats

dependent metabolic pathways. The findings may be interpreted as a first indication that this agent is involved in the development of salt-dependent blood pressure elevation.

Inhibition of CYP-450 dependent metabolism of arachidonic acid with ABT blocked, at least initially, the usual increase in SBP, however, the blockade started to disappear after day 5 of the diet. The ABT dependent reduction in blood pressure was absent or minor when measured on day 21 of exposure to HS diet (Fig. 4). It is not clear why the effect of ABT treatment was only temporary. It will be recalled that at the dose applied here the drug inhibits mostly the synthesis of 20-HETE, a potent vasoconstrictor, with little effect on EETs. In experiments with longer ( 21 days') exposure to high-salt diet we saw that ABT increased renal medullary NO. This could depend on elimination of the effect of 20-HETE, the product of CYP-450 enzyme which normally competes with nitric oxide synthase (NOS) for the heme molecule; such a mechanism was supported by earlier findings [32, 33]. However, the enhancement of NO, intrarenal and probably also systemic, was, apparently, not sufficient to cause a significant and sustained reduction in total peripheral vascular resistance or blood pressure.

An increase in tissue bioavailability of EETs after $c$-AUCB treatment would be expected to cause systemic vasodilation and inhibit distal tubular reabsorption: both actions are potentially antihypertensive $[29,34]$. $c$-AUCB treatment was found to lower BP in rats on standard diet, and in Ren-2 transgenic rat with inducible hypertension [13, 35]. Indeed, it has been suggested that inability to increase the EETs level contributes to the pathogenesis of hypertension [36]. In this study c-AUCB treatment did not attenuate the SBP increase induced by high-salt intake, which does not support the hypothesis that also salt-dependent hypertension might be caused by a deficit of EETs. At least this was not the causal factor with short-term application of high-salt diet.

Taken together, no antihypertensive effect of increasing EETs and some effectiveness of elimination of 20-HETE suggest that it was the latter agent that in the present experiments was involved in the development of sodium dependent blood pressure elevation, at least in the initial phase. For unknown reasons, after a few days' exposure to high-salt diet elimination of the vasoconstrictor effect of 20-HETE by ABT was, apparently, no more an important factor opposing the increase in blood pressure. It can be speculated that elimination by ABT of the usual natriuretic action of 20-HETE promoted progressing fluid retention, a prohypertensive process. This effect should become more important with time and may have offset the direct vasorelaxant effect. Indeed, at the end of salt exposure the renal excretion was the lowest in the ABT-treated group (Table 2). However, in disagreement with the above speculation, this coincided with the MABP value that was the lowest among groups. Possibly, low UNaV was simply the consequence of low MABP ("low pressure antinatriuresis").

High sodium intake and renal vascular responses to ACh and NE: role of CYP-450dependent metabolites of arachidonic acid

Altered renal vascular responsiveness to vasoactive agents could be an index of or even a factor engaged in the development of sodium dependent hypertension. However, in our study the ACh-induced increases in renal total and regional perfusion parameters were similar in rats on standard and HS diet. This indicates that in the renal vascular bed and the present experimental setting, a relatively short-term high sodium intake did not modify the endothelial capacity to release vasodilator NO, and such modification was not involved in the observed progressing increase in blood pressure. Admittedly, the responsiveness to ACh could still be altered in extrarenal vascular beds, which could contribute to blood pressure elevation in HS rats. On the other hand, the baseline medullary NO content was found to be significantly lower in HS compared to STD rats when measured after 3 weeks' exposure to high-salt diet (Fig. 4). In earlier studies application of high-sodium diet for 6-weeks' was shown to cause a $40 \%$ reduction of renal vasodilator effects of exogenous acetylcholine [37], which suggests that sufficient duration of the exposure is needed for deterioration of the response. 


\section{Kidney Blood Pressure Research}

Kidney Blood Press Res 2015;40:323-334

\begin{tabular}{l|l}
\hline DOI: $10.1159 / 000368508$ & (C) 2015 S. Karger AG, Basel
\end{tabular}

Published online: May 31, 2015

www.karger.com/kbr

Similarly as with Ach, HS diet did not consistently modify the renal haemodynamic responses to NE, with one exception. The expected decreases in total renal blood flow (RBF) and cortical perfusion (CBF) were associated with some tendency to a decrease in OMBF on standard diet, but this contrasted with a puzzling increase in OMBF in HS rats (Fig. 2). Remarkably, the baseline value was very low in this group which showed salt-dependent BP increase (Table 2); this observation fits the concept that medullary hypoperfusion may promote blood pressure elevation [18]. Nevertheless, the reason for the paradoxical NEinduced OMBF increase is unclear. Earlier studies of the effects of HS diet on vasoconstrictor responses to NE yielded contradictory results: both increases [38] or no influence on vascular responses [39] were reported.

A striking observation was that in rats on high salt intake inhibition of CYP-450 with ABT, leading most probably to depletion of elevated 20-HETE, virtually abolished renal vascular dilatation in response to exogenous ACh. It is not clear why maintaining the influence of this agent was necessary for ACh-induced vasodilation, as observed in untreated HS rats. It can be speculated that in the absence of the vasoconstrictor 20-HETE the available NO and EETs caused close-to-maximal systemic and renal vasodilatation, reflected by the blood pressure level that was the lowest among groups. Therefore, further stimulation of NO release by ACh did not cause any further substantial decrease in the vascular tone.

Unlike with Ach, our present study failed to disclose consistent effects of changing the activity of 20-HETE or EETs on renal vascular responses to NE.

\section{Conclusion}

In summary, compared with the vast evidence on the role of the RAS in the development of salt-dependent hypertension [7], the available data supporting the involvement of CYP450-dependent active metabolites of AA are not entirely conclusive. Nonetheless, we showed in this study that normotensive rats without overt salt sensitivity showed a progressive increase in blood pressure during 10-days' exposure to high salt diet. The finding that the diet increased renal generation of 20 -HETE (as indicated by increasing urinary concentration) and that blood pressure elevation during HS intake was at least temporarily inhibited by blockade of 20-HETE synthesis, strongly suggest that this vasoconstrictor agent was at least in part responsible for salt-induced blood pressure increase.

The reactivity of renal vessels to exogenous vasoactive agents was not altered during this short-term exposure to high salt diet. When the exposure was prolonged to 21 days, reduced bioavailability of NO was probably a factor involved in sustained salt-dependent elevation of blood pressure.

\section{Disclosure Statement}

The authors of this manuscript state that they do not have any conflict of interests and nothing to disclose.

\section{Acknowledgements}

This work was supported by the Polish Ministry of Science and Higher Education (Grant: N N401 225634). L.Č. was supported by a grant of the Ministry of Health within the project for the development of research organization 00023001 (IKEM) - institutional support.

B.D.H. was supported in part by NIEHS grant ES02710, NIEHS Superfund grant P42 ES04699, NIHLB grant HL059699, and Grant Number U54 NS079202 awarded to B.D.H. B.D.H. is a George and Judy Marcus Senior Fellow of the American Asthma Foundation. 


\section{Kidney \\ Blood Pressure Research}

Kidney Blood Press Res 2015;40:323-334

DOI: $10.1159 / 000368508$

Published online: May 31, 2015

(C) 2015 S. Karger AG, Basel

www.karger.com/kbr

We are greatly indebted to dr. Piotr Tomaszewski and dr. Grażyna Kubiak-Tomaszewska from the Department of Biochemistry and Clinical Chemistry, Pharmaceutical Faculty, Medical University of Warsaw, for their help with measurement of 20-HETE in urine samples.

\section{References}

1 Varagic J, Ahmad S, Brosnihan KB, Varagic J, Ahmad S, Brosnihan KB, Habibi J, Tilmon RD, Sowers JR, Ferrario CM: Salt-induced renal injury in spontaneously hypertensive rats: effects of nebivolol. Am J Nephrol 2010;32:557-566.

2 He FJ, MacGregor GA: A comprehensive review on salt and health and current experience of worldwide salt reduction programmes. J Hum Hypertens 2009;23:363-384.

3 He FJ, MacGregor GA: Reducing population salt intake worldwide: from evidence to implementation: Prog Cardiovasc Dis 2010;52:263-282.

4 Brown IJ, Tzoulaki I, Candeias V, Elliott P: Salt intakes around the world: implications for public health. Int J Epidemiol 2009; 38:791-813.

5 Boegehold MA: The effect of high salt intake on endothelial function: reduced vascular nitric oxide in absence of hypertension. J Vasc Res 2013;50:458-467.

6 Park JS, Kim S, Jo CH, Oh IH, Kim GH: Effects of dietary salt restriction on renal progression and interstitial fibrosis in adriamycin nephrosis. Kidney Blood Press Res 2014;39:86-96.

7 Drenjančević-Perić I, Jelaković B, Lombard JH, Kunert MP, Kibel A, Gros M: High-salt diet and hypertension: focus on the renin-angiotensin system. Kidney Blood Press Res 2010;34:1-11.

8 Wu H, Liang Y, Zheng Y, Bai Q, Zhuang Z, A L, Zheng D, Wang Y: Up-regulation of intrarenal renin-agiotensin system contributes to renal damage in high-salt induced hypertension rats. Kidney Blood Press Res 2014;39:526-535.

9 Roman RJ: P-450 metabolites of arachidonic acid in the control of cardiovascular function. Physiol Rev 2002;82:131-185.

10 Oyekan AO, Youseff T, Fulton D, Quilley J, McGiff JC: Renal cytochrome P450 $\omega$-hydroxylase and epoxygenase activity are differentially modified by nitric oxide and sodium chloride. J Clin Invest 1999;104:1131-1137.

11 Capdevila JH, Falck JR, Imig JD: Role of the cytochrome P450 arachidonic acid monooxygenases in the control of systemic blood pressure and experimental hypertension. Kidney Int 2007;72:683-689.

12 Hye Khan MA, Neckár J, Manthati V, Errabelli R, Pavlov TS, Staruschenko A, Falck JR, Imig JD: Orally active epoxyeicosatrienoic acid analog attenuates kidney injury in hypertensive Dahl salt-sensitive rat. Hypertension 2013;62:905-913.

13 Honetschlägerová Z, Z Husková Z, Vaňourková Z, Sporková A, Kramer HJ, Hwang SH, Tsai HJ, Hammock BD, Imig JD, Červenka L, Kopkan L: Renal mechanisms contributing to the antihypertensive action of soluble epoxide hydrolase inhibition in Ren-2 transgenic rats with inducible hypertension. J Physiol 2011;589:20719.

14 Capdevila JH, Wei S, Yan J, Karara A, Jacobson HR, Falck JR, Guengerich FP, DuBois RN: Cytochrome P-450 arachidonic acid epoxygenase. Regulatory control of the renal epoxygenase by dietary salt loading. J Biol Chem 1992;267:21720-21726.

15 Kagota S, Tamashiro A, Yamaguchi Y, Nakamura K, Kunitomo M: High salt intake impairs nitric vascular oxide/cyclic guanosine monophosphate system in spontaneously hypertensive rats. J Pharmacol Exp Ther 2002;302:344-351.

16 Zhu J, Mori T, Huang T, Lombard JH: Effect of high salt diet on NO release and superoxide production in rat aorta. Am J Physiol Heart Circ Physiol 2004;286:H575-H583.

17 Lenda DM, Boegehold MA: Effect of a high salt diet on microvascular antioxidant enzymes. J Vasc Res 2002;39:41-50.

18 Sofola O, Knill A, Myers D, Hainsworth R, Drinkhill M: High-salt diet and responses of the pressurized mesenteric artery of the dog to noradrenaline and acetylcholine. Clin Exp Pharmacol Physiol 2004;31:696699. 


\section{Kidney \\ Blood Pressure Research}

Kidney Blood Press Res 2015;40:323-334

DOI: $10.1159 / 000368508$

Published online: May 31, 2015

(C) 2015 S. Karger AG, Basel

www.karger.com/kbr

19 Lenda DM, Sauls BA, Boegehold MA: Reactive oxygen species may contribute to reduced endotheliumdependent dilation in rats fed high salt. Am J Physiol Heart Circ Physiol 2000;279:H7-H14.

20 Su P, Kaushal KM, Kroetz DL: Inhibition of renal arachidonic acid $\omega$ - hydroxylase activity with ABT reduces blood pressure in the SHR. Am J Physiol Regul Integr Comp Physiol 1998;275:R426-438.

21 Maier KG, Henderson L, Narayanan J, Alonso-Galicia M, Falck JR, Roman RJ: Fluorescent HPLC assay for 20-HETE and other P-450 metabolites of arachidonic acid. Am J Physiol Heart Circ Physiol 2000;279:H863-H871.

22 Cowley AW Jr: Role of the renal medulla in volume and arterial pressure regulation. Am J Physiol Regul Integr Comp Physiol 1997;273:R1-R15.

23 Hwang SH, Tsai HJ, Liu JY, Morisseau C, Hammock BD: Orally bioavailable potent soluble epoxide hydrolase inhibitors. J Med Chem 2007;50:3825-3840.

24 Zhang X, Broderick M: Amperometric detection of nitric oxide. Modern Aspects Immunobiol 2000;1:160165.

25 Grzelec-Mojzesowicz M, Sadowski J: Renal tissue NO and intrarenal haemodynamics during experimental variations of NO content in anaesthetised rats. J Physiol Pharmacol 2007;58:149-163.

26 Ying WZ, Sanders PW: Dietary salt increases endothelial nitric oxide synthase and TGF-beta1 in rat aortic endothelium. Am J Physiol 1999;277:H1293-1298.

27 Dobrowolski L, Kompanowska-Jezierska E, Walkowska A, Sadowski J: Sodium intake determines the role of adenosine A2 receptors in control of renal medullary perfusion in the rat. Nephrol Dial Transplant 2007;22:2805-2809.

28 Dobrowolski L, Walkowska A, Kompanowska-Jezierska E, Kuczeriszka M, Sadowski J: Effects of ATP on rat haemodynamics and excretion: role of sodium intake, nitric oxide and cytochrome P450. Acta Physiol (Oxf) 2007;189:77-85.

29 Imig JD: Epoxides and soluble epoxide hydrolase in cardiovascular physiology. Physiol Rev 2012;92:101130 .

30 Oyekan 0: Differential effects of 20-hydroxyeicosatetraenoic acid on intrarenal blood flow on the rat. J Pharmacol Exp Ther 2005;313:1289-1295.

31 Wang J, Roman RJ, Falck R, de la Cruz L, Lombard JH: Effects of high-salt diet on CYP450-4A hydroxylase expression and active tone in mesenteric resistance arteries. Am J Physiol Circ Physiol 2005;288:H15571565.

32 Fleming I: Cytochrome P450 enzymes in vascular homeostasis. Circ Res 2001;89:753-762.

33 Kuczeriszka M, Bądzyńska B, Kompanowska-Jezierska E: Cytochrome P-450 monooxygenases in control of renal haemodynamics and arterial pressure in anaesthetized rats. J Physiol Pharmacol 2006;57:179-185.

34 Li J, Carroll MA, Chander PN, Falck JR, Sangras B, Stier CT: Soluble epoxide hydrolase inhibitor, AUDA, prevents early salt-sensitive hypertension. Front Biosci 2008;13:3480-3487.

35 Sporková A, Kopkan L, Varcabová S, Husková Z, Hwang SH, Hammock BD, Imig JD, Kramer HJ, Cervenka L: Role of cytochrome P-450 metabolites in the regulation of renal function and blood pressure in 2-kidney 1-clip hypertensive rats. Am J Physiol Regul Integr Comp Physiol 2011;300:R1468-R1475.

36 Imig JD: Epoxide hydrolase and epoxygenase metabolites as therapeutic targets for renal disease. Am J Physiol Renal Physiol 2005;289:F496-F503.

37 Fiore MC, Jimenez PM, Cremonezzi D, Juncos LI, García NH: Statins reverse renal inflammation and endothelial dysfunction induced by chronic high salt intake. Am J Physiol Renal Physiol 2011;301:F263-F270.

38 Soltis EE, Bartrug BL, Newman PS: Chronic hypertension and vascular alterations following short-term dietary salt intake in postweanling rats. Dev Pharmacol Ther 1993;20:107-110.

39 Weber DS, Frisbee JC, Lombard JH: Selective potentiation of angiotensin-induced constriction of skeletal muscle resistance arteries by chronic elevations in dietary salt intake. Microvasc Res 1999;57:310-319. 